\title{
What makes lung cancer invade?
}

\author{
Victor A. Ferraris, MD, PhD
}

See related article on pages 1404-14.

Invasion is a critical step in lung tumor progression. The interaction between tumor cells and their surroundings plays an important role in tumor invasion and metastasis. Critical questions exist regarding mechanisms of invasion. The article by Wang and coauthors in this issue of the Journal reports their approach to this subject with advanced molecular techniques. They used next-generation sequencing of micro-RNAs (miRNAs) to develop a comprehensive profile of miRNAs in squamous cell cancer of the lung (SCCL). They identified miRNA signatures that may provide biomarkers for early detection of SCCL and may generate subclassifications of tumors with unique therapeutic targets. This article gives readers an insight into the complexity of miRNA sequencing and functional mapping. The topic is important, but complex and esoteric. I believe that thoracic surgeons need to have a working knowledge of this subject, and this article introduces this subject in a very comprehensive and scientifically rigorous manner. Time spent understanding this subject will ultimately prove valuable as new molecular treatments of this dismal disease appear.

It helps to understand the methods used in the analysis of Wang and coauthors, because many of the terms in the article are not familiar to most readers. MicroRNAs (miRNAs) are endogenous noncoding small RNAs of about 18 to 25 nucleotides. Mature miRNAs are highly conserved RNA molecules that can regulate the development and progression of lung cancer by promoting the expression of oncogenes or by inhibiting tumor suppressor genes. Wang and coauthors used next-generation sequencing to characterize the miRNA transcriptome (meaning "all miRNA in a pooled tissue") of SCCL tumor tissue, surrounding lung tissue, and normal lung tissue from pooled patient samples. For small RNA targets, such as miRNA, the RNA is isolated through size selection. This can be

\footnotetext{
From the Division of Cardiothoracic and Vascular Surgery, University of Kentucky, Lexington, Ky.

Disclosures: Author has nothing to disclose with regard to commercial support.

Received for publication Feb 10, 2015; accepted for publication Feb 12, 2015; available ahead of print March 20, 2015.

Address for reprints: Victor A. Ferraris, MD, PhD, Division of Cardiothoracic and Vascular Surgery, University of Kentucky, A301 Kentucky Clinic, 740 S Limestone, Lexington, KY 40536-0284 (E-mail: ferraris@uky.edu).

J Thorac Cardiovasc Surg 2015;149:1415-6

$0022-5223 / \$ 36.00$

Copyright (c) 2015 by The American Association for Thoracic Surgery http://dx.doi.org/10.1016/j.jtcvs.2015.02.026
}

performed with a size-exclusion gel, through size-selection magnetic beads, or with commercially developed kits, as used by Wang and coauthors. Once isolated, linkers are added to the $3^{\prime}$ and $5^{\prime}$ ends of the miRNA, and linked miRNA is then purified. The ligation step adds DNA adaptors to both ends of the small RNAs, which act as primer binding sites during reverse transcription and polymerase chain reaction amplification. The final step is complement DNA (cDNA) generation through reverse transcription and subsequent amplification of the cDNA into macro amounts by means of quantitative polymerse chain reaction. These steps describe the part labeled "Library and Sequencing" in Figure 1 in the article of Wang and coauthors. Subsequent steps shown in their Figure 1 describe next-generation sequencing (labeled "Data Analysis"), and at the crux of this analysis is a process named miRDeep2. ${ }^{1}$ miRDeep2 is a program that maps sequences of known miRNAs to the samples being studied. The trimmed reads of miRNA were input into miRDeep2 to quantify known miRNAs against a searchable database of published miRNA sequences (miRBase) and predict novel miRNAs (http://sourceforge.net/projects/mireap/). The terms in this paragraph do not roll off the tongue of thoracic surgeons with any regularity, but they are a good starting point for understanding this study.

Vast amounts of data are presented in the subsequent figures in the article of Wang and coauthors, and the article tries to condense the findings into manageable subsets. It is unreasonable to expect only a few significant results when dealing with the large data sets represented by the sample preparations of Wang and coauthors. The average reader has a nagging concern when reading an article like this. Because the information presented is not routine and is not well understood by the average reader, the exact importance and context of the findings are a little bit suspect, and conclusions are obscure. There are some criticisms that the reviewers raise, but they are mostly abstruse and are the comments of someone who is familiar with the subject matter. Critiques might include something like "converting RNA into cDNA by means of reverse transcriptase can introduce biases and artifacts that may interfere with both the proper characterization and quantification of transcripts, especially in miRNAs with low expression." A counter to this statement would be that the accuracy of the steps outlined in Figure 1 of the article of Wang and coauthors, including the use of 
miRDeep2, is greater than $98 \%$. There may be some missed miRNAs that have functional importance, but the results shown in Figures 2 and 3 in the article of Wang and coauthors have face validity and provide a starting point for further in-depth investigations.

Perhaps the most important part of the article is the ability to tie the changes in miRNA to physiologic processes. Wang and coauthors call this "[p]rediction and enrichment analysis of target genes." The miRNAs expressed differentially between cancer cells and normal or nearly normal tissues were hybridized to genes to identify genes that might be important for invasion of tumor cells into surrounding tissues. The genes discovered by this process can be fitted into physiologic processes that may serve as therapeutic targets (Figure 6 and
Table 1 in the article of Wang and coauthors). Further studies that use these potential targets may provide unique therapies for SCCL. It is worth reading this article to the very end, because only then does it become clearer why one would even bother delving into this mysterious information. It is not hard to envision a day when the molecular genetics and the regulatory role of miRNAs are routine topics for discussion of lung cancer treatment. Thoracic surgeons need to know this information and be able to converse reasonably with peers about its implications.

\section{Reference}

1. Friedländer MR, Mackowiak SD, Li N, Chen W, Rajewsky N. miRDeep2 accurately identifies known and hundreds of novel microRNA genes in seven animal clades. Nucleic Acids Res. 2012;40:37-52. 